\title{
Guanylic nucleotide starvation affects Saccharomyces cerevisiae mother-daughter separation and may be a signal for entry into quiescence
}

\author{
Isabelle Sagot*, Jacques Schaeffer and Bertrand Daignan-Fornier
}

\author{
Address: Institut de Biochimie et Génétique Cellulaires, UMR CNRS 5095 - Université Victor Segalen / Bordeaux II 1, rue Camille Saint Saëns - F- \\ 33077 Bordeaux Cedex - France \\ Email: Isabelle Sagot* - Isabelle.Sagot@ibgc.u-bordeaux2.fr; Jacques Schaeffer - Jacques.Schaeffer@ibgc.u-bordeaux2.fr; Bertrand Daignan- \\ Fornier - B.Daignan-Fornier@ibgc.u-bordeaux2.fr \\ * Corresponding author
}

Published: 04 May 2005

BMC Cell Biology 2005, 6:24 doi:10.1 186/147|-2121-6-24
Received: 18 February 2005

Accepted: 04 May 2005

This article is available from: http://www.biomedcentral.com/I47|-2/2I/6/24

(C) 2005 Sagot et al; licensee BioMed Central Ltd.

This is an Open Access article distributed under the terms of the Creative Commons Attribution License (http://creativecommons.org/licenses/by/2.0), which permits unrestricted use, distribution, and reproduction in any medium, provided the original work is properly cited.

\begin{abstract}
Background: Guanylic nucleotides are both macromolecules constituents and crucial regulators for a variety of cellular processes. Therefore, their intracellular concentration must be strictly controlled. Consistently both yeast and mammalian cells tightly correlate the transcription of genes encoding enzymes critical for guanylic nucleotides biosynthesis with the proliferation state of the cell population.
\end{abstract}

Results: To gain insight into the molecular relationships connecting intracellular guanylic nucleotide levels and cellular proliferation, we have studied the consequences of guanylic nucleotide limitation on Saccharomyces cerevisiae cell cycle progression. We first utilized mycophenolic acid, an immunosuppressive drug that specifically inhibits inosine monophosphate dehydrogenase, the enzyme catalyzing the first committed step in de novo GMP biosynthesis. To approach this system physiologically, we next developed yeast mutants for which the intracellular guanylic nucleotide pools can be modulated through changes of growth conditions. In both the pharmacological and genetic approaches, we found that guanylic nucleotide limitation generated a mother-daughter separation defect, characterized by cells with two unseparated daughters. We then showed that this separation defect resulted from cell wall perturbations but not from impaired cytokinesis. Importantly, cells with similar separation defects were found in a wild type untreated yeast population entering quiescence upon nutrient limitation.

Conclusion: Our results demonstrate that guanylic nucleotide limitation slows budding yeast cell cycle progression, with a severe pause in telophase. At the cellular level, guanylic nucleotide limitation causes the emergence of cells with two unseparated daughters. By fluorescence and electron microscopy, we demonstrate that this phenotype arises from defects in cell wall partition between mother and daughter cells. Because cells with two unseparated daughters are also observed in a wild type population entering quiescence, our results reinforce the hypothesis that guanylic nucleotide intracellular pools contribute to a signal regulating both cell proliferation and entry into quiescence. 


\section{Background}

Guanylic nucleotides are critical for multiple crucial cellular processes such as replication, transcription, translation and signalization via small GTPases. Most cell types recycle GMP from guanosine or guanine, which are either taken up from the surrounding environment or synthesized by the intracellular metabolism. GMP can also be synthesized de novo from IMP via two consecutive enzymatic steps, both highly conserved through evolution. Initially, the inosine monophosphate dehydrogenase (IMPDH) catalyzes conversion of IMP into XMP, the first committed step in de novo GMP biosynthesis. Subsequently, GMP synthetase converts the newly produced XMP into GMP.

The expression of IMPDH encoding genes is tightly regulated. In both yeast and mammalian cells, high guanylic nucleotide levels repress the transcription of IMPDH encoding genes [1,2]. More strikingly, the transcription of IMPDH encoding genes in mammals is linked to cellular proliferation. In non-dividing cells, the expression level of IMPDHs is low, whereas it is highly increased in actively proliferating cells such as cancerous cells [3-6]. Further, IMPDH over-expression bypasses the anti-proliferative effect of p53, indicating that this p53 function requires proper control of IMPDH activity [7].

Saccharomyces cerevisiae cells have two major sources of guanylic nucleotides: intracellular IMP and extracellular guanine. Therefore GMP synthesis fully relies on IMPDH and GMP synthetase activities in the absence of guanine in the growth medium. Consistently, mutations in these cognate genes lead to guanine auxotrophy $[8,9]$. Similar to mammalian cells, $S$. cerevisiae genes involved in GMP biosynthesis are highly expressed during exponential growth, but are actively repressed through specific regulatory sequences when cells arrest proliferation upon nutrient limitation [10]. Moreover, the intracellular GTP/GDP ratio drastically decreases when yeast cells enter a quiescent state [11]. These experiments lead to the appealing hypothesis that intracellular guanylic nucleotides levels contribute to a signal regulating cell proliferation. However, the molecular pathways linking cell cycle progression to IMPDH activity, and thus to intracellular guanylic nucleotide pools remain unknown.

Mycophenolic acid (MPA) is a well-characterized noncompetitive and reversible inhibitor of $\mathrm{IMPDH}$ that severely depletes intracellular GTP pool down to $10 \%$ of normal level $[12,13]$. MPA reduces or even abrogates proliferation of various cell types [14,15]. MPA particularly affects lymphocyte division and because it has few secondary effects, its pro-drug form, the mycophenolate mofetil (Cellcept, Roche), is in path to replace cyclosporine A as a commonly used immunosuppressive drug. At the cellular level, MPA inhibits lymphocyte cell cycle progression by arresting cells in G1. Although this effect correlates with the depletion of guanylic nucleotide pools $[13,16]$, addition of guanosine and 8-aminoguanosine, which can partially replenish guanylic nucleotide pools, does not allow MPA treated cells to re-enter G2/M [17]. Thus, MPA treatment, although apparently blocking cells in G1, could also affect later steps of the cell cycle. In budding yeast, MPA treatment slows cell proliferation and causes various effects on gene expression and thus on the yeast proteome [18]. In a previous study, we have shown that MPA affects yeast cell size, DNA content, budding pattern and causes occasional perturbations of actin and microtubule cytoskeletons [18]. In addition, several mutants affected for various cellular functions are hypersensitive to MPA [19]. However, these data do not point at an obvious molecular process that would account for the effects of MPA on cell proliferation.

Here, to gain insight into the molecular relationships between intracellular guanylic nucleotide levels and cell cycle progression, we studied the effects of MPA treatment on Saccharomyces cerevisiae cell cycle progression. We first demonstrated that, although cells did not arrest in a particular cell cycle stage (confirming that there was no checkpoint for guanylic nucleotides in yeast), a large proportion of the population was slowed in telophase. We further observed that many MPA treated cells presented two unseparated daughter cells. We have shown that this specific morphology was due to a defect in mother-daughter separation and that it was probably a consequence of cell wall perturbations. To validate the results obtained with MPA, we developed yeast mutants in which guanylic nucleotide pools could be modulated by the composition of the growth medium. Using these genetic tools, we confirmed that cell separation was indeed the cell cycle step mostly perturbed by guanylic nucleotides starvation. Finally, the observation that cells entering quiescence also displayed the characteristic "two-daughter cells" morphology strongly suggests that a decrease in intracellular guanylic nucleotide levels may be part of a signal for yeast cells to enter stationary phase.

\section{Results \\ Mycophenolic acid treatment particularly affects the last step of the yeast cell cycle}

MPA treatment affects yeast growth in a concentrationdependent manner [18]. This growth defect can result from either a general slowing down of the entire cell cycle or a pause in a specific cell cycle step. To gain insight into this issue, wild type yeast cells were synchronized in G1 using alpha factor and released in either the absence or the presence of $100 \mu \mathrm{g} / \mathrm{mL}$ of MPA. At such a concentration, MPA does not affect cell viability nor totally arrest cell growth [18]. Higher MPA concentration gave similar 


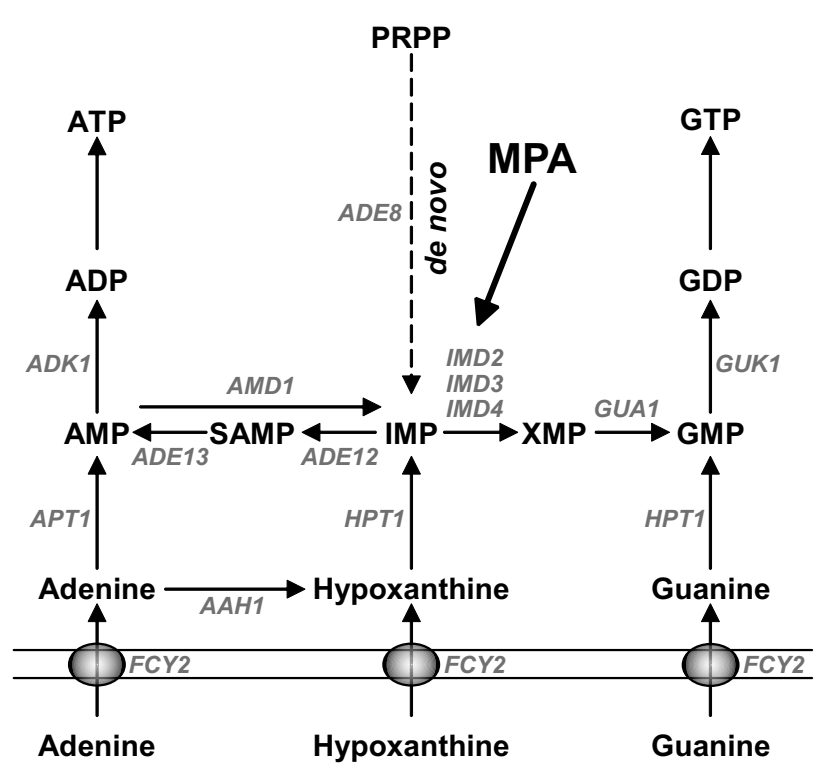

Figure I

Schematic representation of the purine nucleotide synthesis pathway in yeast. The solid thin lines represent the plasma membrane. ADP: adenosine-5'-diphosphate; AMP: adenosine-5'-monophosphate; ATP: adenosine-5'-triphosphate; GDP: guanosine-5'-diphosphate; GMP: guanosine-5'monophosphate; GTP: guanosine-5'-triphosphate; IMP: inosine-5'-monophosphate; PRPP: 5-phosphoribosyl-I-pyrophosphate; SAMP: S-adenosine-5'-monophosphate; XMP: xanthosine-5'-monophosphate. Genes are shown in grey italic font and encode the following enzymatic activities: $A A H I$ : adenine deaminase; $A D E 8$ : 5'-phosphoribosylglycinamide formyltransferase; $A D E / 2$ : adenylosuccinate synthetase; $A D E$ I3: adenylosuccinate lyase; $A D K$ I: AMP kinase; $A M D$ I: AMP deaminase; APTI: adenine phosphoribosyltransferase; FCY2: purine cytosine permease; GUA I: GMP synthetase; GUKI: GMP kinase; HPTI: hypoxanthine-guanine phosphoribosyltransferase; IMD2, IMD3, and IMD4: IMP dehydrogenases (IMDI is not indicated here because it is not expressed and is thought to be a pseudogene). Mycophenolic acid (MPA) inhibits IMP dehydrogenases.

effects, most probably because yeast cells detoxified the drug [19]. We then monitored cell cycle progression by FACS. As shown in figure 2A, although progression through initial cell cycle steps was slower for MPA treated cells, the population lagged most predominantly in a $2 \mathrm{~N}$ DNA content stage. Fluorescence microscopy revealed that although for MPA-treated cells the anaphase onset was slightly delayed, its duration was almost similar to the anaphase of control cells (Fig. 2B). In fact, treated cells were mostly pausing in a stage where the DNA masses were totally separated (telophase, Fig. 2C). To our sur- prise, in the treated population we observed mother cells with two apparent daughter cells (Fig. 2D and 2E). The cells with two apparent daughters represented more than $50 \%$ of the population 300 minutes after the release from G1. To confirm this result, a non-synchronized population was treated with MPA. After 4 hours in the presence of MPA, more than $30 \%$ of the budding cells displayed two apparent daughters (Fig. 3), a phenotype we refer to as "bibudded", for simplicity.

A "bibudded" phenotype could result either from a single mother cell with two daughter cells, or from an unrelated G1 cell "sticking" to a normally budding cell. Since MPA treated cells were found to be sensitive to sonication, we used a fluorescence-based approach to distinguish between these two possibilities. Equal amounts of cells over-expressing a green variant of GFP were mixed with cells over-expressing a blue variant of GFP, grown to $\mathrm{OD}_{600 \mathrm{~nm}} 0.2$ and then treated with MPA.

After 4 hours of incubation, less than $1 \%(0.3 \% \pm 0.3$, $\mathrm{N}>100$ for each of 4 independent experiments) of "bibudded" cells displayed one apparent daughter expressing a different GFP variant than its joined budding cell. Thus, more than $99 \%$ of the cells with two apparent daughters were monocolor, which is far more than the $50 \%$ expected for a random population of false "bibudded" cells. This experiment demonstrated that the population is indeed composed of a large proportion of cells with two attached daughters. In conclusion, MPA treatment particularly slowed down telophase and caused the appearance of cells with two unseparated daughters.

\section{MPA effects are reversed by extracellular guanine and are not a consequence of translation inhibition}

To generate guanylic nucleotides, the requirement for IMPDH activity can be bypassed by the addition of guanine into the growth medium (Fig. 1). To show that the effects of MPA on cell cycle progression specifically result from a decrease of intracellular guanylic nucleotide pools, we treated cells with MPA in guanine-supplemented growth medium. In these conditions, only 3\% of the cells displayed two unseparated daughters (Fig. 3) and no growth defect was detected (data not shown and [18]).

As $100 \mu \mathrm{g} / \mathrm{mL}$ MPA causes a significant decrease of the translation efficiency [18], we considered the possibility that "bibudded" cells result from impaired translation. To this end, we examined cells treated for 4 hours with the general translational inhibitor cycloheximide at a concentration that abolished translation [20]. In contrast to MPA-treated cells, cycloheximide-treated cells did not exhibit more than one bud $(0.8 \% \pm 0.3 \%$ of the budding cells had two buds, N>200). Therefore, by decreasing intracellular guanylic nucleotide pools, MPA treatment 


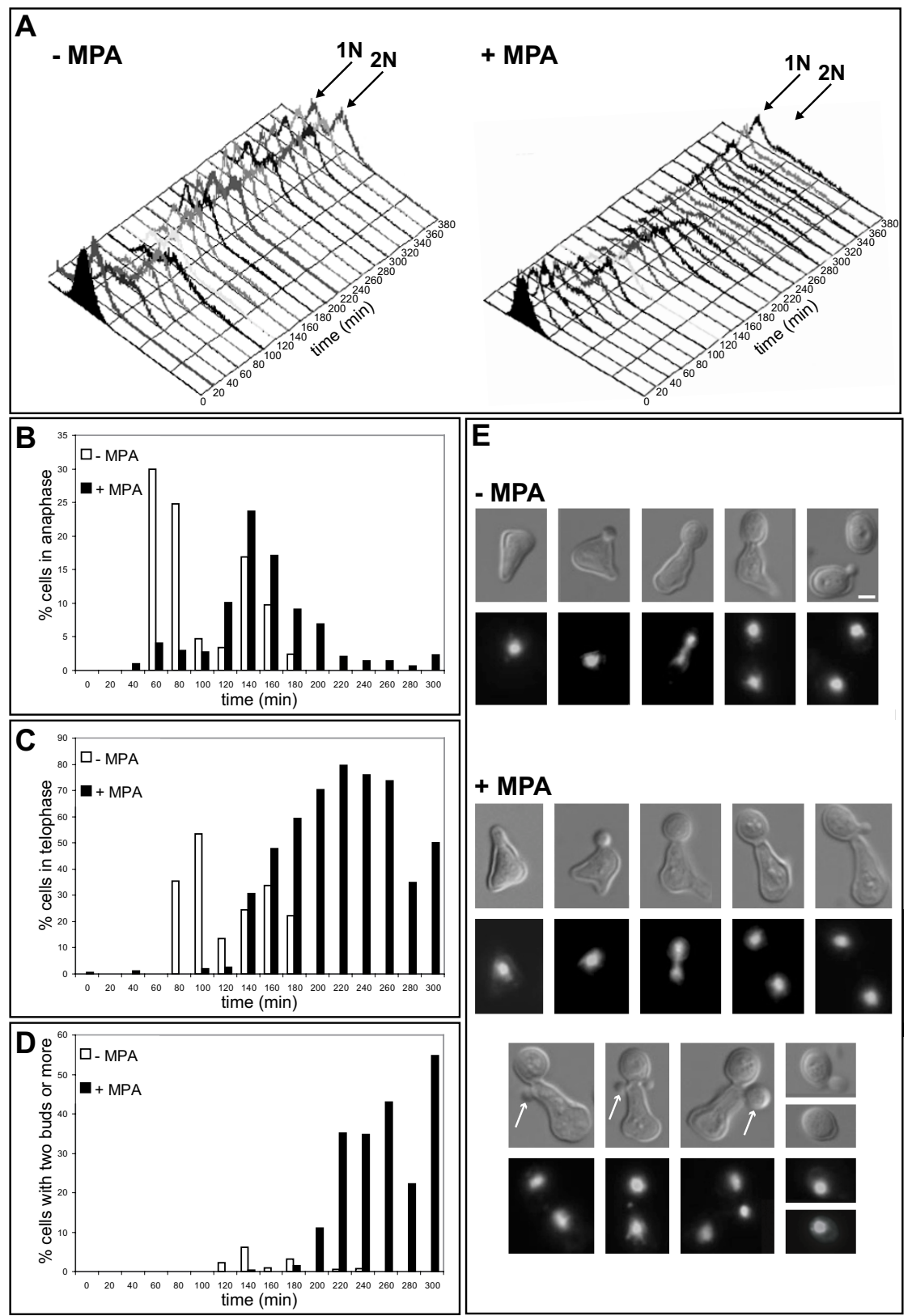

Figure 2

Effects of MPA on the yeast cell cycle progression. A. Cell cycle progression of a yeast cell population synchronized in GI with alpha factor and released in the absence (left) or in the presence of $100 \mu \mathrm{g} / \mathrm{mL}$ MPA (right) analyzed by FACS. B. Percentage of cells in anaphase in function of the progression through the cell cycle. Cells are the same as in A. More than 200 cells were counted for each time point. Cells were scored as being in anaphase when the mother cell DNA mass was clearly still connected to the DNA mass of the daughter cell, typically as the third cell shown in the - MPA panel of figure 2E. C. Percentage of cells in telophase in function of the progression through the cell cycle. Cells are the same as in A. More than 200 cells were counted for each time point. Cells were scored as being in telophase when displaying two clearly separated DNA masses, typically as the fourth cell shown in the - MPA panel of figure 2E. D. Percentage of cells with two or more daughter cells in function of the progression through the cell cycle. Cells are the same as in A. More than 200 cells were counted for each time point. E. Cells representative of each cell cycle stage (phase contrast and propidium iodide staining of the nucleus) for the untreated (top panel) or MPA treated (bottom panel) population. The unusual elongated cell morphology is due to the alpha factor treatment. Arrows indicate examples of second daughter cell appearing while the first daughter cell is not yet separated from the mother cell. Bar: $2 \mu \mathrm{m}$. 


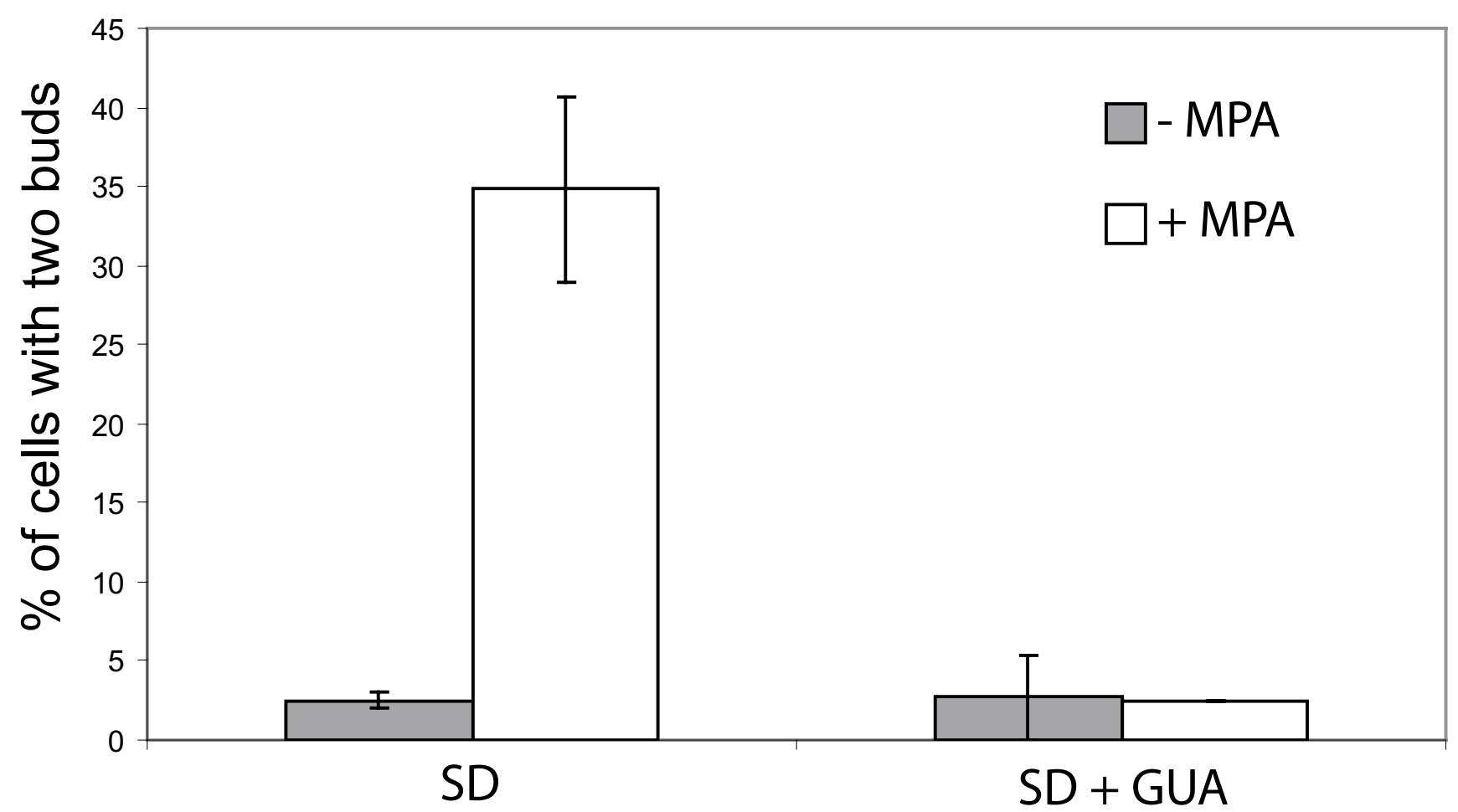

Figure 3

Effects of MPA treatment on an unsynchronized yeast cell population. Percentage of budding cells with two daughter cells after 4 hours growth in SD medium without (left) or with guanine (right) in the absence (grey bars) or in the presence (white bars) of $100 \mu \mathrm{g} / \mathrm{mL}$ MPA. More than 200 cells were counted for each condition.

caused a slowing down of the yeast cell cycle progression, the telophase being mostly affected. Consequently, "bibudded" cells accumulate and this, independently of MPA effects on translation efficiency.

\section{Mutations affecting the guanylic nucleotide biosynthesis cause the emergence of cells with two unseparated daughters}

Although addition of guanine to the growth medium fully reversed the effects of MPA, pharmacological studies have the caveat of possible secondary targets. Thus, we developed mutant yeast strains in which the guanylic nucleotide pools can be modulated by a simple change of the growth conditions. Because of genetic redundancy in IMPDH encoding genes, no single imd mutant is auxotroph for guanine, we therefore chose to use a mutant in the GUA1 gene which encodes GMP synthetase, the enzyme converting XMP into GMP (Fig. 1). A gua1s mutant is unable to synthesize guanylic nucleotides in the absence of guanine in the growth medium. To compare the effects of guanylic nucleotide starvation with the depletion of another purine, we combined the gua1 $\Delta$ deletion with the ade8 $\Delta$ deletion that leads to adenine auxotrophy. The double mutant ade $8 \Delta$ gua $1 \Delta$ is thus auxotroph for both guanine and adenine (Fig. 4A). When both guanine and adenine were provided in the growth medium, the double mutant ade8s gua1s grew like the isogenic ade $8 \Delta$ control strain and, the ade $8 \Delta$ gua1s population contained less than $3 \%$ of "bibudded" cells (Fig. $4 \mathrm{~A}$ and $4 \mathrm{~B})$. After a 4-hour shift to a growth medium lacking guanine, $20 \%$ of the cells displayed two unseparated daughter cells. By contrast, shifting the cells to growth medium lacking adenine did not induce the emergence of "bibudded" cells (Fig. 4B). Therefore, "bibudded" cells appearance is specific to a guanylic nucleotide starvation.

In order to diminish the intracellular guanylic nucleotide pools by another route, we constructed an ah $1 \Delta$ amd1s double mutant. The AAH1 gene encodes adenine aminohydrolase, an enzyme converting adenine into hypoxanthine. The AMD1 gene encodes the AMP deaminase enzyme, which converts AMP into IMP (Fig. 1). The de 
A

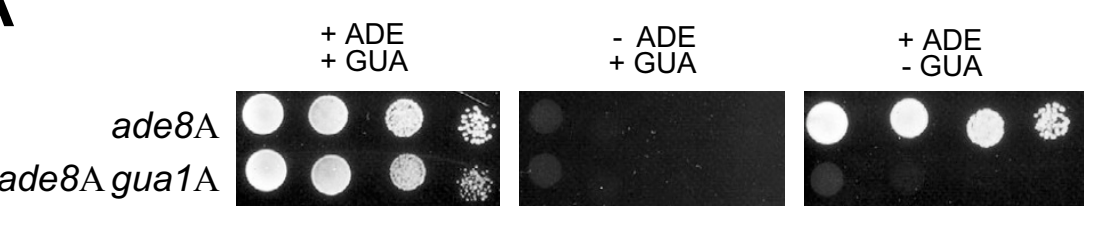

B

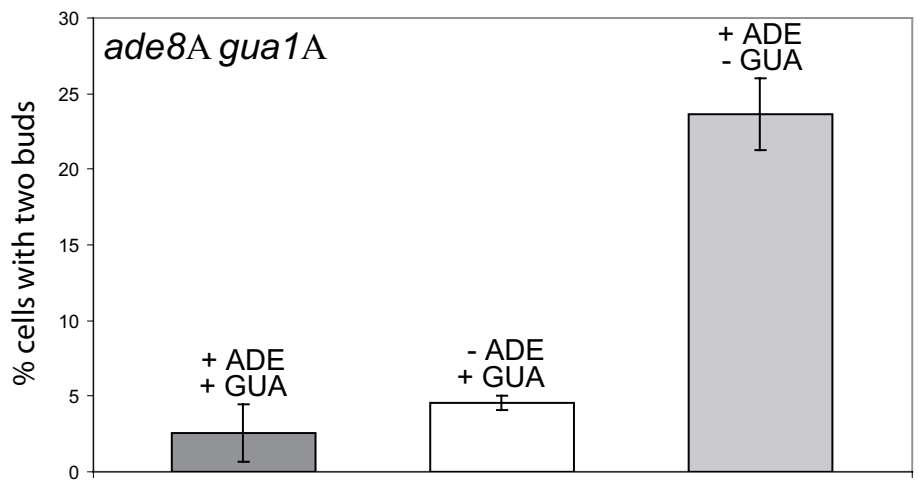

C

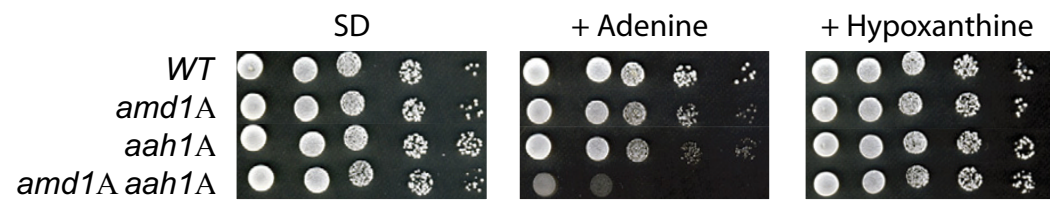

D

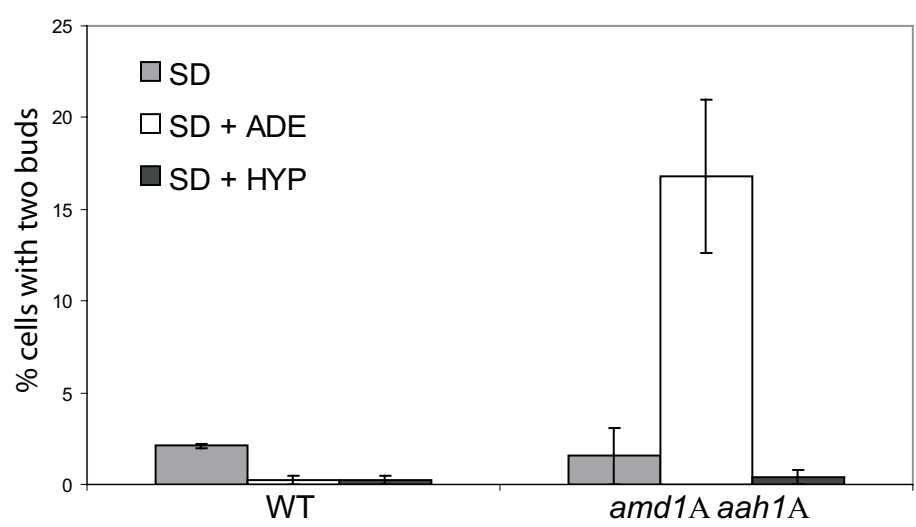

Figure 4

Effects of guanylic nucleotide starvation using mutants of the purine nucleotide biosynthesis pathway. A. Growth of an ade $8 \Delta$ gual $\Delta$ double mutant and an isogenic ade $8 \Delta$ single mutant on SD medium containing the indicated purines. The SD + ADE + GUA medium contains $25 \%$ Adenine / 75\% Guanine ratio (total purine concentration of $0.3 \mathrm{mM}$ ). B. The ade $8 \Delta$ gual $\Delta$ strains was grown in SD medium containing $25 \%$ Adenine $/ 75 \%$ Guanine ratio to $\mathrm{OD}_{600 \mathrm{~nm}}=0.2 ;$ then shifted into the indicated medium. After 4 hours of incubation at $30^{\circ} \mathrm{C}$, the percentage of cells with two daughters among budding cells was counted. More than 200 cells were counted for each condition. C. Growth of the WT strain, isogenic single amd Is or aah $I \Delta$ mutant strains and amd $I \Delta$ aah I $\Delta$ double mutant strain on SD medium, SD medium supplemented with adenine or SD medium supplemented with hypoxanthine. D. WT strain (left) and amd I $\Delta$ aah I $\Delta$ double mutant strain (right) were grown in $\mathrm{SD}$ medium to $\mathrm{OD}_{600 \mathrm{~nm}}=0.2$ and then shifted into the indicated medium. After 4 hours of incubation at $30^{\circ} \mathrm{C}$, the percentage of cells with two daughters among budding cells was counted. More than 200 cells were counted for each condition. 
novo synthesis of IMP from PRPP is inhibited by the presence of adenine in the growth medium [21]. Consequently, in the presence of adenine, the aah1 $1 \Delta$ amd1s double mutant has theoretically no path to synthesize GMP, neither from the de novo pathway nor from adenine. Accordingly, without extracellular guanine (or hypoxanthine), the guanylic nucleotide pools of the aah1 $\Delta$ amd1 $\Delta$ double mutant cannot be replenished and its growth is strongly affected (Fig. 4C). The aah1s amd1s double mutant was grown in SD medium without purine to $\mathrm{OD}_{600 \mathrm{~nm}}=0.2$ and then shifted into a medium containing adenine as a sole source of purine. After 4 hours, the percentage of cells with two unseparated buds was counted. As shown in figure $4 \mathrm{D}$, "bibudded" cells only arose when the aah1 $\Delta$ amd1 $1 \Delta$ double mutant cells were grown in a medium containing adenine. Thus, modifying the guanylic nucleotide pools through mutations in the purine nucleotide biosynthesis pathway provoked the same effect that MPA treatment on the yeast cell cycle: drastically impaired cell separation without cell cycle arrest, resulting in cells with two unseparated daughters.

\section{Guanylic nucleotide starvation does not affect the formation of cellular structures required for completion of cytokinesis}

The decrease of intracellular guanylic nucleotide pools caused the appearance of cells with two unseparated buds. We further characterized these abnormal cells to identify the cellular process(es) critically impaired by this starvation and thus likely responsible for this particular phenotype.

When synchronized cells were treated with MPA, the timing of emergence and the size of the second bud compared to the first one (see Fig. 2E) strongly suggested that MPA treated cells started a new cell cycle before the separation of the first daughter cell. Cells with two daughters follow a normal budding pattern (Fig. 2E and 5A), and are morphologically very different from the multibudded cells observed for polarity mutants impaired in bud emergence. Thus, the decrease of intracellular guanylic nucleotide pools did not likely affect cell polarity establishment. Further, most of the cells with two buds presented wild type, polarized actin patches and cables (Fig. 5A, middle lane), confirming previous studies showing that MPA treatment does not drastically affect the actin cytoskeleton [18]. In addition, nuclei of the "bibudded" cells are properly positioned (Fig. 5A, bottom lane) although abnormal mitosis occurs in less than $5 \%$ of the cells (Fig. 5A, + MPA right panel). Thus, polarization establishment and nuclear segregation were not drastically affected in cells with two unseparated daughters.

Microscopic observation of synchronized MPA-treated cells revealed that although anaphase on-set was delayed, its duration was almost similar to the anaphase in control cells (Fig. 2B). Therefore, MPA treatment did not critically affect the mitotic exit network (MEN). Further, MPA treatment did not disrupt proper localization of Tem $1 \mathrm{p}$, a RAS GTPase governing the MEN, neither the localization of its GTP exchange factor (GEF) Lte1p (Fig. 5B, left and middle panels). Taken together, these results suggest that MPA treatment does not significantly perturb anaphase progression, and consequently does not activate the anaphase checkpoint.

Because MPA severely affected progression through telophase, we examined the formation of cellular structures essential for the completion of cytokinesis. Figure 5A shows that both the first and the second daughter cell can build an actin cytokinetic ring. Further, more than $95 \%$ of the MPA treated cells $(\mathrm{N}>200)$ maintain proper localization of the septin Cdc12p (Fig. 5B, right panel), suggesting strongly that MPA-induced guanylic nucleotide pools depletion does not affect the septin ring formation and stability. Moreover, in "bibudded" cells, the septin ring can split to allow acto-myosin ring contraction for both the first and the second daughter cell (Fig. 5B, right panel). Therefore, guanylic nucleotide starved cells displayed all the structures required for cytokinesis completion, and the cellular processes leading to the motherdaughter separation defect must occur later in the cell cycle.

\section{Guanylic nucleotide starvation affects cell wall separation} As cells with two unseparated daughters properly form both actin and septin rings, we examined whether the cytoplasm was still continuous between the first bud and its mother. After a 4 hour MPA treatment, we digested the cell wall with zymolyase and counted the number of remaining "bibudded" cells. Figure 6A shows that mild treatment with zymolyase cause a decrease of the "bibudded" cell number suggesting strongly that in those cells, the cytoplasm is no more continuous between the mother cell and at least one daughter cell. Thus, guanylic nucleotide depletion apparently did not affect cytoplasm constriction but rather a later step in the daughter cell separation process. To confirm this result, we observed MPA-treated cells by electron microscopy after Thiéry coloration, which reveals polysaccharides and therefore the cell wall. As shown in figure $6 \mathrm{~B}$, the cytoplasm between the mother and the first daughter cell is no longer continuous. Thus, guanylic nucleotide starvation did not affect cytoplasm closure but a later step in the daughter cell separation process. Further, the secondary septum of the first daughter cell appears normal by electron microscopy, although some small lacunae were occasionally observed (see inset of Fig. 6B). Therefore, we speculate that reduction of intracellular guanylic nucleotide pools by 
A

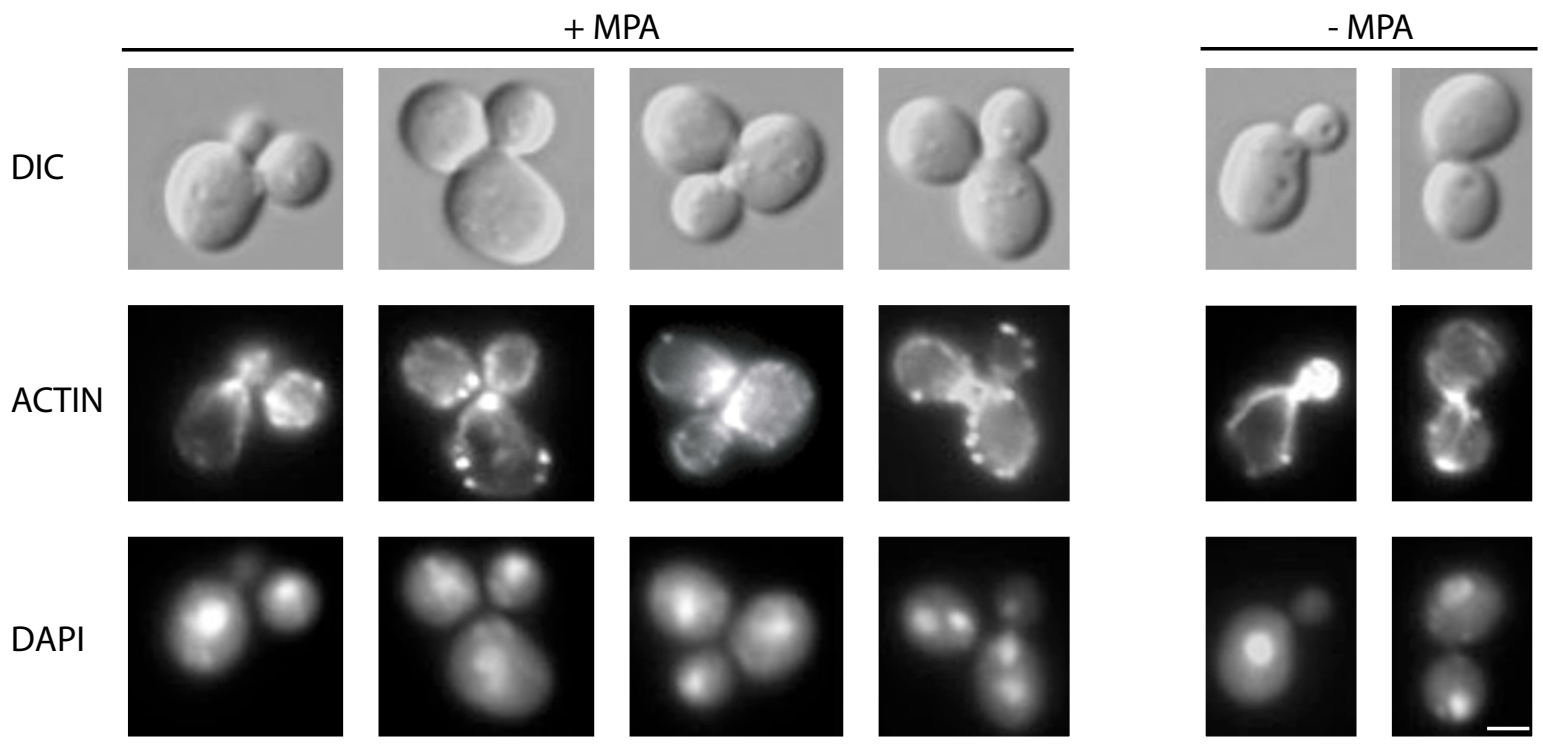

B
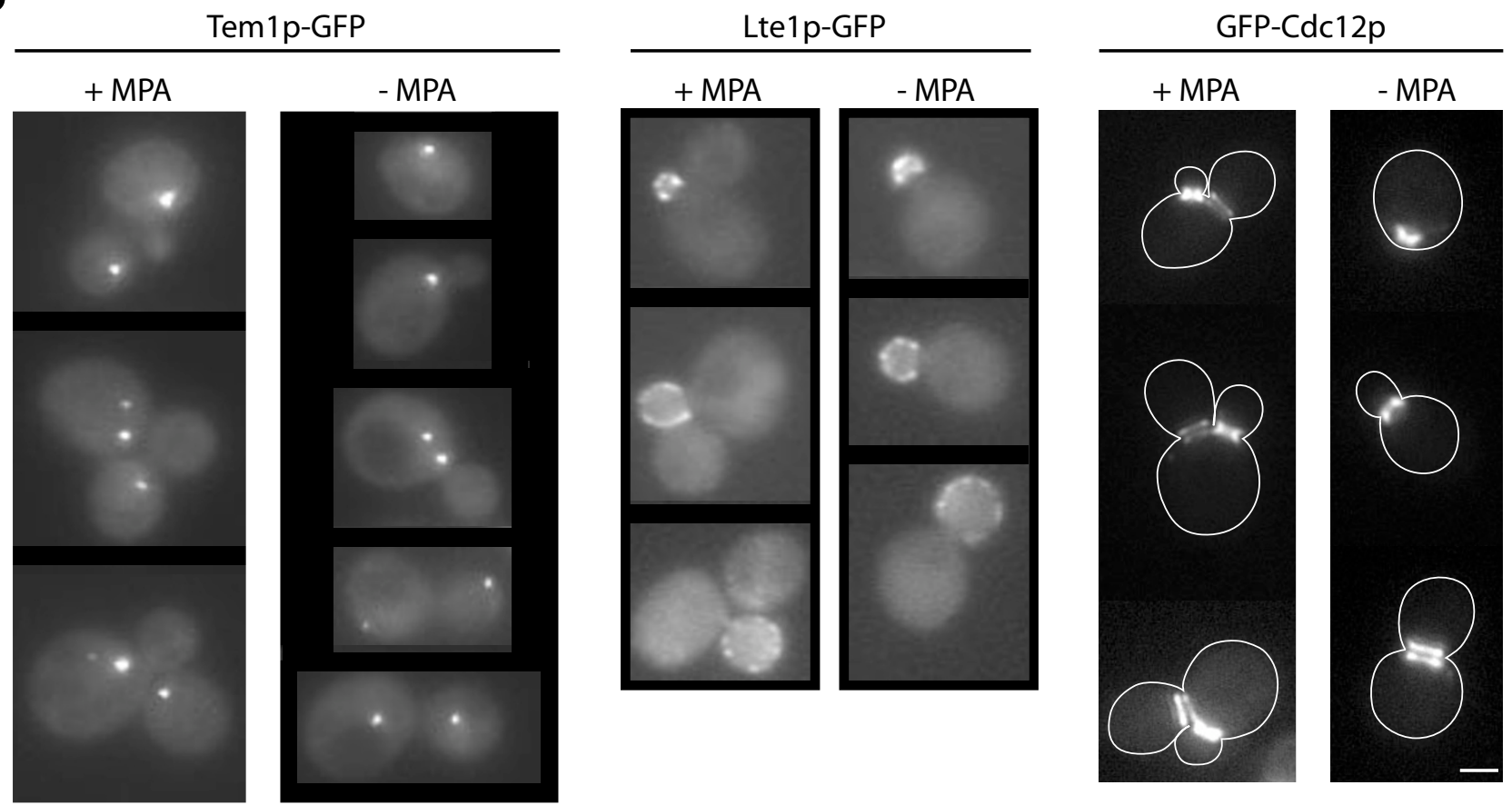

Figure 5

Localization of several cellular structures or fusion proteins in cells with two daughters. A. Cells were untreated (right panel) or treated (left panel) with $100 \mu \mathrm{g} / \mathrm{mL}$ MPA for 4 hours. DIC (top lane), actin Alexa-phalloidin staining (middle lane) and DAPI staining (bottom lane) are shown. B. Localization of endogenous Tem Ip-GFP (left panel), endogenous Ltel pGFP (middle panel) and GFP-Cdc 12p expressed from a centromeric plasmid under the control of its own promoter (right panel) in cells untreated (right of each panel) or treated for 4 hours with $100 \mu \mathrm{g} / \mathrm{mL}$ MPA (left of each panel). Only cells displaying two buds are shown in the case of MPA treatment. Bar: $2 \mu \mathrm{m}$. 
MPA treatment particularly impinges on the separation of the daughter cell by affecting the cell wall digestion.

\section{A large proportion of cells entering quiescence exhibit two daughters}

In the course of our study, we noticed that a small but reproducible amount of untreated wild type cells displayed two unseparated buds (see for example Fig. 3), a morphology identical to MPA-treated "bibudded" cells. We then examined the frequency of "bibudded" cells during the growth of a wild type yeast population. To our surprise, whereas less than $2 \%$ of the cells with two unseparated buds could be observed during exponential phase, when yeast approached the diauxic shift, more than $30 \%$ of the budding population presented two apparent daughter cells (Fig. 7). This observation suggested a slowing of daughter cell separation upon the last divisions before stationary phase. Like previously, we verified the authenticity of the "bibudded" phenotype by mixing two populations of cells, each expressing one different GFP variant. When this mixed population reached the diauxic shift, we counted the number of cells with two apparent daughters and examined them by fluorescence microscopy. Similar to our results for MPA-treated cells, only $0.9 \% \pm 0.7 \%$ of the apparent "bibudded" cells were bicolor (more than 200 "bibudded" cells were counted for each experiment). Thus, we concluded that those cells were indeed cells with two daughters. This was further supported by observation of those cells by electron microscopy (not shown). We obtained similar results with both the BY4742 and the FL100 genetic background (data not shown) demonstrating that the appearance of cells with two daughters is not specific to the BY4742 background. In conclusion, when a yeast culture approaches stationary phase, a significant proportion of cells behaves like cells in which the intracellular guanylic nucleotide pools have been depleted and give rise to cells with two unseparated daughters.

\section{Discussion}

Guanylic nucleotides are not only "building blocks" for nucleic acids but are also crucial for the regulation of many cellular processes such as G-proteins based signaling pathways. Therefore, cells must maintain their concentrations at a critical level. However, the molecular relationships between intracellular guanylic nucleotide levels and cell proliferation crucial events remain poorly understood.

Here, we have demonstrated that MPA treatment does not cause a firm cell cycle arrest in yeast. Treated cells continue to proliferate, although at a reduced rate (this study and [18]). Further, we have shown that conditional mutants unable to synthesize guanylic nucleotides do not arrest in a particular stage of the cell cycle. Therefore, our results establish that there is no guanylic nucleotide checkpoint is $S$. cerevisiae. By contrast, in mammalian cells, it was previously shown that MPA treatment cause an arrest of cellular proliferation but no guanylic nucleotide specific checkpoint has clearly been identified. Several reports have described that MPA treatment affects mammalian cells ability to commit into division by blocking the transition from G0 to the $S$ phase of the cell cycle $[13,17,22]$. However, if MPA is added when cells have already entered the $S$ phase, the cell cycle arrest occurs in G2/M [22]. Besides, the MPA-induced arrest is not fully reversed by the replenishment of guanylic nucleotide pools $[13,17,22]$. Here, we have shown that even if MPA treatment slows all stages of the yeast cell cycle progression, the most affected step is the mother-daughter cell wall separation, giving rise to "bibudded" cells. This result supports our previous observation that MPA treatment leads to the appearance of many cells with $3 \mathrm{~N}$ DNA content [18]. Importantly, MPA treatment has the same effects on both non-synchronized and synchronized cells treated with the drug upon release from G1 (Fig. 2) or upon release from G2/M after nocodazole synchronization (I. S., B. D.-F. unpublished results). Therefore, unlike in mammals, in yeast, MPA treatment causes the same effects whatever the cell cycle stage of the cells at the time of drug addition. Further, our analysis of mutants demonstrates that the mother-daughter separation defect results solely from guanylic nucleotide pools depletion and is independent of potential MPA secondary targets.

In yeast, the fact that a guanylic nucleotides starvation causes a mother-daughter separation defect was unexpected. Indeed, one could have intuitively supposed that this depletion would rather provoke a drastic defect during DNA replication upon $S$ phase or disturb cell cycle steps for which GTPase driven molecular processes are essential. In fact, upon MPA treatment, cells can still build a daughter cell and cell polarity was found even less affected in this study than in our earlier work [18]. Daughter cell appears to grow normally, mitosis proceeds unperturbed, and mother-daughter closure is properly achieved. Thus, guanylic nucleotides starvation does not critically affect the functions of key GTP binding proteins, such as Cdc42p, Tem1p, tubulin and septins. In this study we observed that in the "bibudded" cells, placement of the second daughter cell properly follows the axial budding pattern of haploid cells, suggesting that the bud-site selection machinery is properly located. In contrast, prolonged MPA treatment (48 hours) leads to a random budding pattern [18]. Thus, long-term guanylic nucleotides starvation may have more drastic effects. Most importantly, our experiments show that complete mother-daughter separation is not required for the mother cell to pass through START and to generate a second daughter cell (Fig. 2E). Therefore, our data confirm that no additional checkpoint 


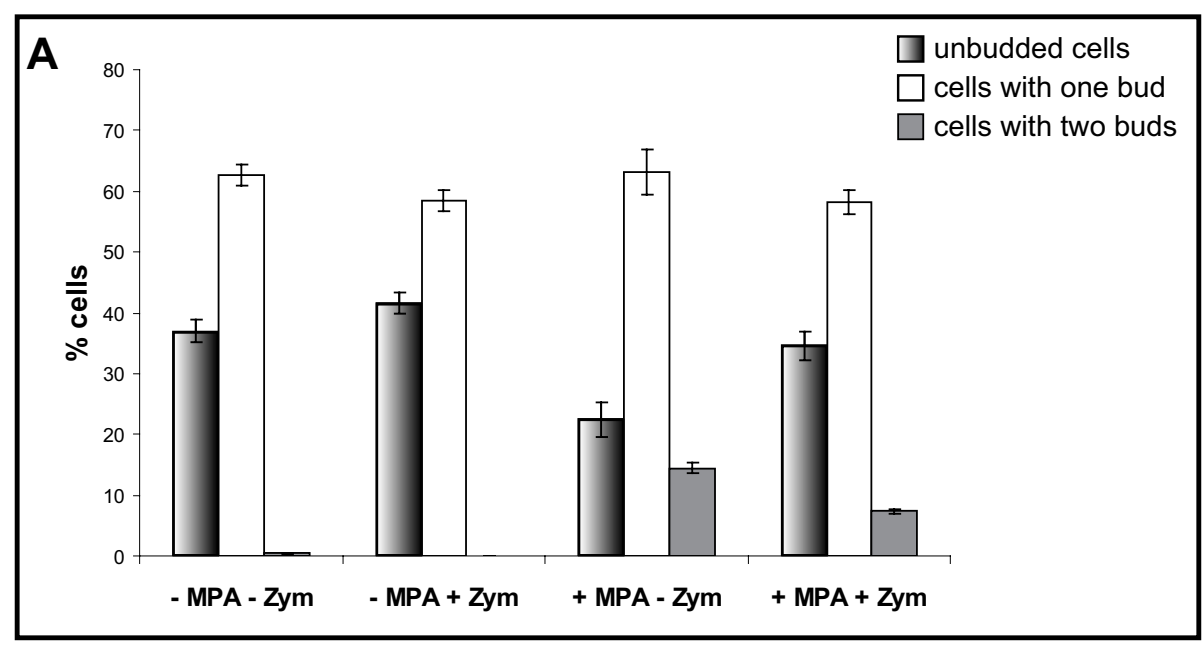

B

+ MPA
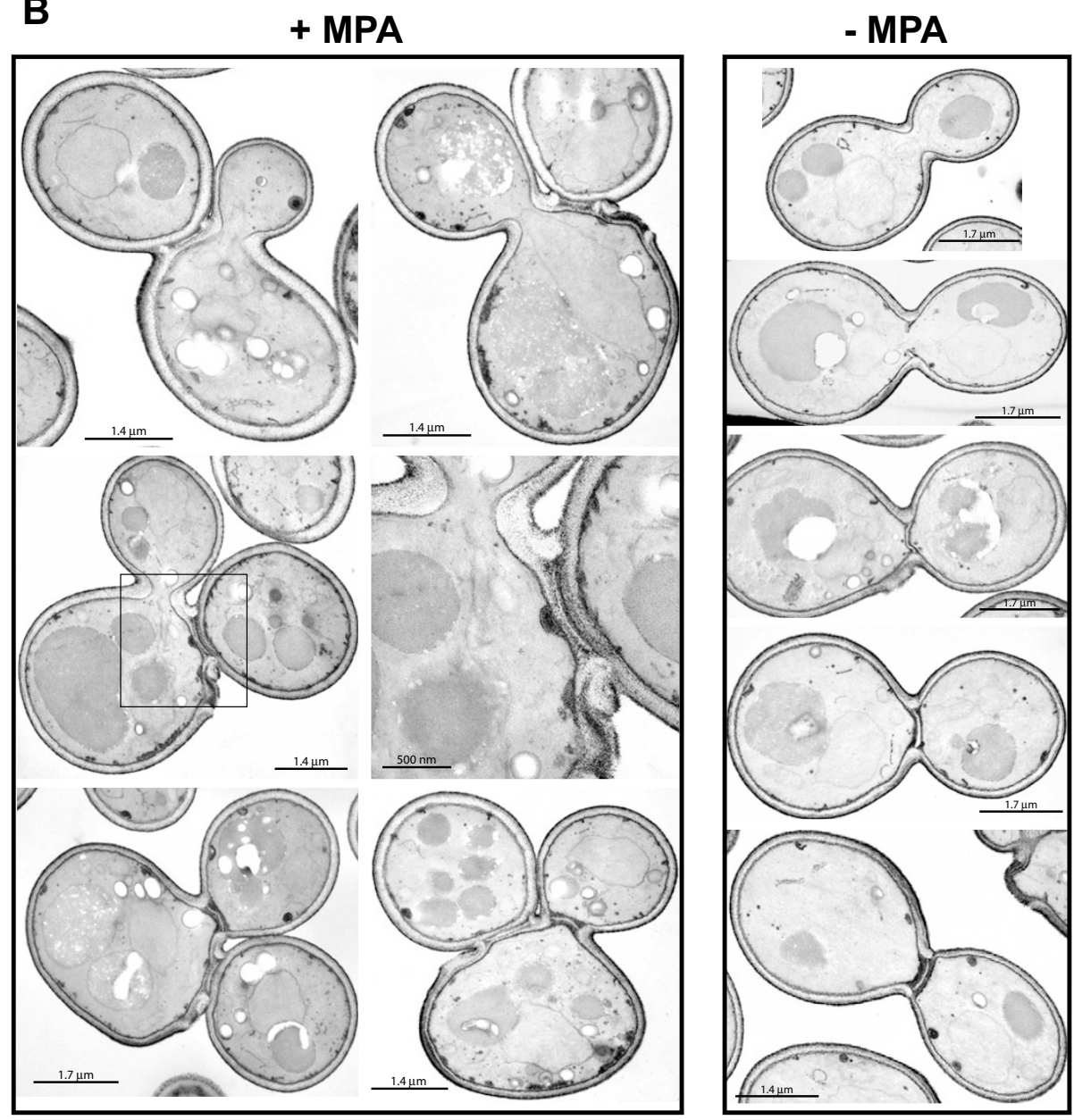

Figure 6

Effects of MPA on the yeast cell wall. A. Percentage of unbudded (shadow bars), single budded (white bars) and of cells with two buds (grey bars) after 4 hours treatment with $100 \mu \mathrm{g} / \mathrm{mL}$ MPA followed by a mild digestion of the cell wall with zymolyase. More than 200 cells were counted for each condition. B. Electron microscopy pictures of cells with two buds obtained after 4 hours treatment with $100 \mu \mathrm{g} / \mathrm{mL}$ MPA. Untreated control cells are shown on the right panel each steps of the septum formation are illustrated (from top to bottom). 


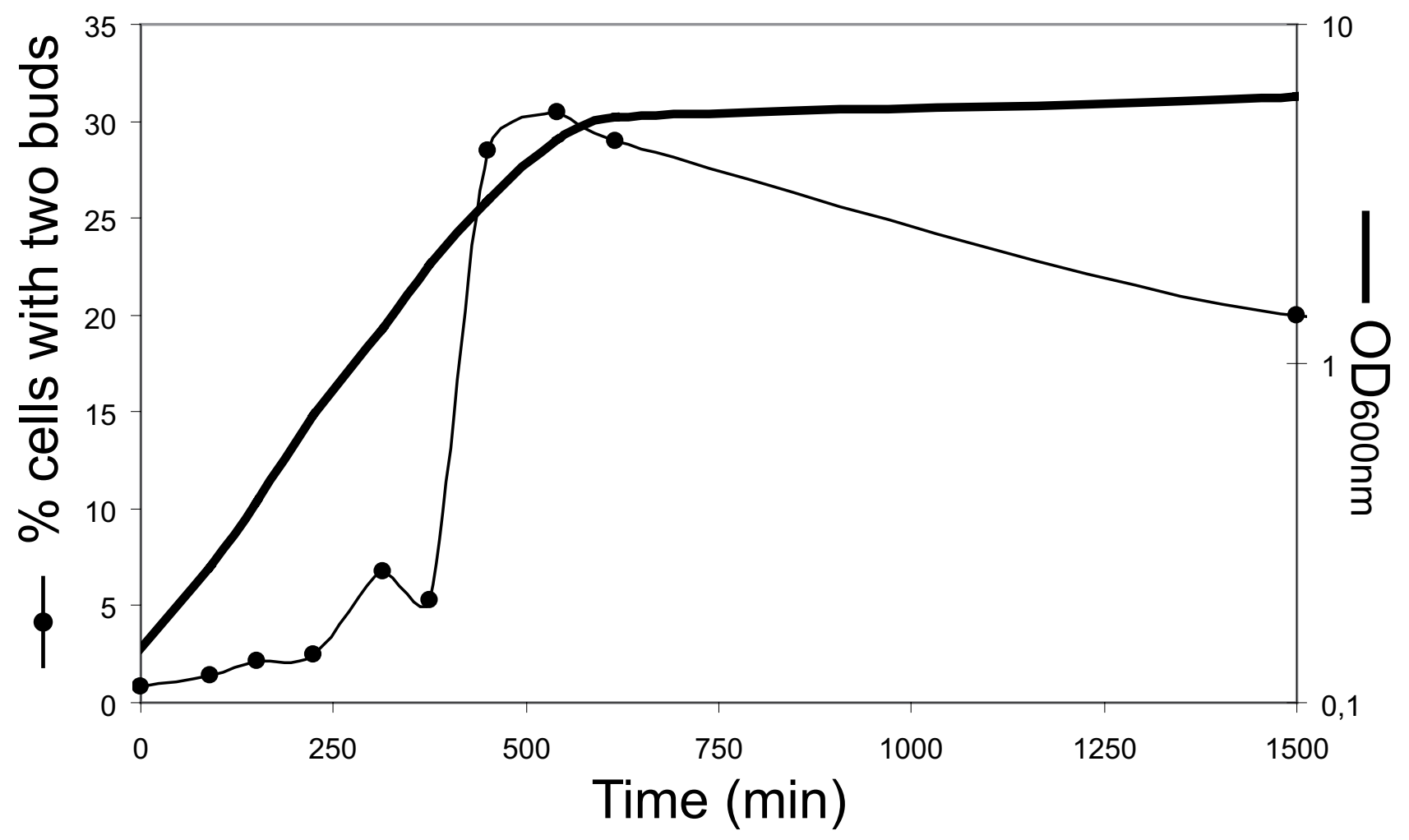

Figure 7

Number of cells with two daughters as a function of the age of the culture. WT cells were grown in SC medium and the percentage of cells with two daughters among budding cells as a function of time was counted (black circles). For each time point, more than 300 cells were counted. $\mathrm{OD}_{600 \mathrm{~nm}}$ is indicated as a black bold line.

blocks the cell cycle progression when anaphase is properly achieved.

What molecular targets trigger the mother-daughter separation defect upon guanylic nucleotide starvation? Observation of "bibudded" cells by electron microscopy revealed no obvious defect in the overall septum architecture of abnormally unseparated daughter cell. Nevertheless, MPA treatment increases cells sensitivity to SDS [19], zymolyase or sonication (I. S., B. D.-F. unpublished results), strongly suggesting cell wall defects. In fact, previous works have illustrated links between guanylic nucleotides metabolism and cell wall integrity, particularly through the synthesis of mannoproteins, essential components of the fungal cell wall. GDP-mannose is the common substrate for mannosyltransferases, enzymes catalyzing the addition of mannose residues on mannoproteins core oligosaccharides. In budding yeast, the GDP-mannose pyrophosphorylase Psa1p is an essential enzyme that synthesizes GDP-mannose, using GTP as a substrate. It was previously shown that MPA treatment affects Psa1p expression [18] and that Psa1p depletion leads to cell separation failure [23]. Further, Shimma et al have demonstrated that the major defect of a guk1 conditional mutant strain, that is impaired for GDP biosynthesis (Fig. 1), was a decrease in GDP-mannose level (to about $25 \%$ of the wild type levels) that leads to mannose outer chain elongation defects [24]. Accordingly, we have observed cell separation defect in guk1 cells (I. S., B. D.-F., unpublished results). In addition, yeast lacking mannosyltransferase encoding genes, such as OCH1, MNN10 or ANP1 display both hypersensitivity to MPA [19] and a cell separation defect similar to the one observed for guanylic nucleotide starved cells [25-27]. Therefore, one major consequence of guanylic nucleotide starvation could be a significant decrease in the GDP-mannose pool that in turn leads to a mother-daughter separation defect.

The most intriguing aspect of the regulation of intracellular guanylic nucleotide pools is the correlation between 
the IMPDH activity and cellular proliferation in mammalians models. Interestingly, the transcription of the IMD2 gene is actively shut off via regulatory sequences when yeast cells enter stationary phase upon nutrients limitation [2]. Transcriptome analyses have demonstrated that AAH1, HPT1 and GUA1 are among the most promptly down regulated genes when nutrients become limiting. Thus, it appears that an entire process is devoted to rapidly decrease the intracellular guanylic nucleotide pools when cells enter stationary phase. Here, we have demonstrated that the typical "bibudded" phenotype obtained during guanylic nucleotide starvation also occurs in untreated wild type cells achieving their last divisions upon nutrients limitation. Thus, an identical morphology is observed for both guanylic nucleotide starvation and entry into quiescence.

Finally, the GTP/GDP ratio is very sensitive to growth conditions, rapidly decreasing during the diauxic shift and drastically dropping upon nutrients starvation. Further, this ratio may regulate RAS GTPases activity by influencing its guanylic nucleotide loading equilibrium [11]. RAS and TOR pathways are key regulators that coordinate yeast proliferation with nutrients availability. Recent work has suggested that the TOR protein acts as ATP sensor in mammals [28]. Thus it is appealing to speculate that in parallel to the TOR pathway, intracellular guanylic nucleotides levels are part of a signal that regulate cell proliferation via the modulation of RAS GTPases activity.

\section{Conclusion}

Using either mycophenolic acid, a molecule that specifically inhibits the first committed step in de novo GMP biosynthesis or mutations in the guanylic nucleotide biosynthesis pathway, we have demonstrated that intracellular guanylic nucleotides limitation causes a motherdaughter cell wall separation defect in budding yeast. This defect leads to the emergence of cells with two unseparated daughters. These "bibudded" cells are also found in a population of cells entering quiescence upon nutrient limitation. These observations further suggest that guanylic nucleotide intracellular pools might contribute to a signal that regulates cell proliferation, particularly upon nutrients limitation and entry into stationary phase.

\section{Methods}

\section{Strains, media and reagents}

Yeast strains used were purchased from Euroscarf (Frankfurt, Germany) and are derivatives of BY4741 or BY4742 [29]. SD and SD casa media were described previously [2] and were supplemented when required with tryptophan $(0.2 \mathrm{mM})$, uracil $(1.8 \mathrm{mM})$, guanine $(0.3 \mathrm{mM})$, adenine $(0.3 \mathrm{mM})$, hypoxanthine $(0.3 \mathrm{mM})$. Cycloheximide was purchased from Sigma and used at $50 \mu \mathrm{g} / \mathrm{mL}$. Mycophe- nolic acid (MPA) was purchased from Amresco, (Ohio, USA) and was used at $100 \mu \mathrm{g} / \mathrm{mL}$.

\section{Plasmids}

pB1594 integrates three tandem copies of GFP at the 3' end of the LTE1 coding region (Lte1p-3xGFP) and is a kind gift of D. Pellman [30]. pB1598 integrates three tandem copies of GFP at the 3 ' end of the TEM1 coding region (Tem1p-3xGFP) and is a kind gift of D. Pellman. pYB407 (GFP-Cdc12p - LEU2 - CEN) is a kind gift of Y. Barral [31]. pVTYS65T (URA3, 2 $\mu$ ) allows the expression of the GFP variant containing the S65T mutant (fluorescence maxima: Ex: $488 \mathrm{~nm}, \mathrm{Em}: 511 \mathrm{~nm}$ ) and has been previously described [32]. pVTYBFP2 allows the expression of a GFP variant containing mutations $\mathrm{F} 64 \mathrm{~L}$, S65T, $\mathrm{Y} 66 \mathrm{H}$ et Y145F which coding sequence has been optimized for expression in yeast (fluorescence maxima: Ex $380 \mathrm{~nm}$, Em $440 \mathrm{~nm}$ see [33]). Details of the mutagenesis and constructions are available upon request.

\section{Cell Biology techniques and Fluorescence Microscopy}

Cells were synchronized by addition of alpha-factor at the final concentration of $10 \mu \mathrm{g} / \mathrm{mL}$ directly into the growth medium. After 3 hours of incubation at $30^{\circ} \mathrm{C}$ with agitation, more than $99 \%$ of the cells were unbudded. Cells were washed twice and released into fresh SD medium containing or not MPA. Propidium iodide (Sigma) staining was performed as described in [34] except that cells were fixed with ethanol 70\%. Alexa-568-Phalloidin (Molecular Probes, Eugene, OR) was used to stain filamentous actin as described previously [35]. Zymolyase 20T (ICN Biomedicals, Costa Mesa, CA) was used at 0.2 $\mathrm{mg} / \mathrm{mL}$ on formaldehyde fixed cells resuspended in PBS. Images were acquired with a Marianas system and analyzed with the Slidebook software (Intelligent Imaging Innovations, Inc. Denver, CO) except for GFP-Cdc12p expressing cells that were imaged with a previously described imaging system [32].

\section{Electron Microscopy}

Cells were grown in SD medium to $\mathrm{OD}_{600 \mathrm{~nm}}=0.2$ at $30^{\circ} \mathrm{C}$. MPA was added to the final concentration of 100 $\mu \mathrm{g} / \mathrm{mL}$ and cells were grown for four more hours. Cells were then fixed with glutaraldehyde and osmic acid, and then stained by the method of Thiéry as previously described [36].

\section{Authors' contributions}

JS carried out the electron microscopy. IS did the other experimental work. BDF and IS conceived and coordinated the studies and drafted the manuscript. All authors read and approved the final manuscript.

\section{Acknowledgements}

The authors thank Dr. Y. Barral and Dr. D. Pellman for kindly providing us plasmids and other reagents. We thank C. Saint-Marc for technical assist- 
ance in constructing and characterizing the ade $8 \Delta$ gual $\Delta$ and amd $I \Delta$ aah $I \Delta$ double mutants. We specially want to express our gratitude to Dr. D. Pellman for having kindly allowed us to use his microscope and to Dr. Z. Storchova for her expertise and precious help for FACs experiments. We also acknowledge B. Pinson and A. Breton for helpful discussion and comments on the manuscript. We are very grateful to J. Moseley and E. Coic for their help in proofreading the manuscript. This work was supported by Grant \# 4749 from The Association pour la Recherche Contre le Cancer.

\section{References}

I. Glesne DA, Collart FR, Huberman E: Regulation of IMP dehydrogenase gene expression by its end products, guanine nucleotides. Mol Cell Biol 1991, I I:5417-25.

2. Escobar-Henriques $M$, Daignan-Fornier $B$ : Transcriptional regulation of the yeast gmp synthesis pathway by its end products. J Biol Chem 2001, 276:1523-30.

3. Konno Y, Natsumeda Y, Nagai M, Yamaji Y, Ohno S, Suzuki K, Weber G: Expression of human IMP dehydrogenase types $I$ and II in Escherichia coli and distribution in human normal lymphocytes and leukemic cell lines. J Biol Chem I99I, 266:506-9.

4. Nagai M, Natsumeda Y, Weber G: Proliferation-linked regulation of type II IMP dehydrogenase gene in human normal lymphocytes and HL-60 leukemic cells. Cancer Res 1992, 52:258-61

5. Zimmermann AG, Gu J], Laliberte J, Mitchell BS: Inosine-5'-monophosphate dehydrogenase: regulation of expression and role in cellular proliferation and $\mathbf{T}$ lymphocyte activation. Prog Nucleic Acid Res Mol Biol 1998, 6 I: 181-209.

6. Collart FR, Chubb CB, Mirkin BL, Huberman E: Increased inosine5 '-phosphate dehydrogenase gene expression in solid tumor tissues and tumor cell lines. Cancer Res 1992, 52:5826-8.

7. Liu Y, Bohn SA, Sherley JL: Inosine-5'-monophosphate dehydrogenase is a rate-determining factor for p53-dependent growth regulation. Mol Biol Cell 1998, 9:।5-28.

8. Dujardin G, Kermorgant M, Slonimski PP, Boucherie $\mathrm{H}$ : Cloning and sequencing of the GMP synthetase-encoding gene of Saccharomyces cerevisiae. Gene 1994, I39: I27-32.

9. Hyle JW, Shaw RJ, Reines D: Functional distinctions between IMP dehydrogenase genes in providing mycophenolate resistance and guanine prototrophy to yeast. J Biol Chem 2003, 278:28470-8.

10. Escobar-Henriques M, Collart MA, Daignan-Fornier B: Transcription initiation of the yeast IMD2 gene is abolished in response to nutrient limitation through a sequence in its coding region. Mol Cell Biol 2003, 23:6279-90.

II. Rudoni S, Colombo S, Coccetti P, Martegani E: Role of guanine nucleotides in the regulation of the Ras/cAMP pathway in Saccharomycescerevisiae. Biochim Biophys Acta 200I, I 538: I8I-9.

12. Qiu Y, Fairbanks LD, Ruckermann K, Hawrlowicz CM, Richards DF, Kirschbaum B, Simmonds HA: Mycophenolic acid-induced GTP depletion also affects ATP and pyrimidine synthesis in mitogen-stimulated primary human T-lymphocytes. Transplantation 2000, 69:890-7.

13. Messina E, Gazzaniga P, Micheli V, Barile L, Lupi F, Agliano AM, Giacomello A: Low levels of mycophenolic acid induce differentiation of human neuroblastoma cell lines. Int J Cancer 2004, I | 2:352-354.

14. Yalowitz JA, Jayaram HN: Molecular targets of guanine nucleotides in differentiation, proliferation and apoptosis. Anticancer Res 2000, 20:2329-38.

15. Morath C, Zeier M: Review of the antiproliferative properties of mycophenolate mofetil in non-immune cells. Int J Clin Pharmacol Ther 2003, $41: 465-9$.

16. Allison AC, Eugui EM: Mycophenolate mofetil and its mechanisms of action. Immunopharmacology 2000, 47:85-II8.

17. Laliberte J, Yee A, Xiong Y, Mitchell BS: Effects of guanine nucleotide depletion on cell cycle progression in human $T$ lymphocytes. Blood 1998, 9 I:2896-904.

18. Escobar-Henriques $M$, Balguerie A Monribot $C$, Boucherie $H$, Daignan-Fornier B: Proteome analysis and morphological studies reveal multiple effects of the immunosuppressive drug mycophenolic acid specifically resulting from guanylic nucleotide depletion. J Biol Chem 2001, 276:46237-42.
19. Desmoucelles C, Pinson B, Saint-Marc C, Daignan-Fornier B: Screening the yeast "disruptome" for mutants affecting resistance to the immunosuppressive drug, mycophenolic acid. J Biol Chem 2002, 277:27036-44

20. Clark-Walker GD, Linnane AW: In vivo differentiation of yeast cytoplasmic and mitochondrial protein synthesis with antibiotics. Biochem Biophys Res Commun 1966, 25:8-I3.

21. Daignan-Fornier B, Fink GR: Coregulation of purine and histidine biosynthesis by the transcriptional activators BASI and BAS2. Proc Natl Acad Sci U S A 1992, 89:6746-50.

22. Quemeneur L, Flacher M, Gerland LM, Ffrench M, Revillard JP, Bonnefoy-Berard N: Mycophenolic acid inhibits IL-2-dependent T cell proliferation, but not IL-2-dependent survival and sensitization to apoptosis. J Immunol 2002, I 69:2747-55

23. Warit S, Zhang N, Short A, Walmsley RM, Oliver SG, Stateva LI: Glycosylation deficiency phenotypes resulting from depletion of GDP- mannose pyrophosphorylase in two yeast species. Mol Microbiol 2000, 36: I I 56-66.

24. Shimma $Y$, Nishikawa A, bin Kassim B, Eto A, Jigami Y: A defect in GTP synthesis affects mannose outer chain elongation in Saccharomyces cerevisiae. Mol Gen Genet 1997, 256:469-80.

25. Chepurnaya OV, Kozhina TN, Peshekhonov VT, Korolev VG: The REC4I gene of Saccharomyces cerevisiae: isolation and genetic analysis. Mutat Res 200I, 486:4I-52.

26. Lee BN, Elion EA: The MAPKKK Ste I I regulates vegetative growth through a kinase cascade of shared signaling components. Proc Natl Acad Sci U S A 1999, 96: I2679-84.

27. Mondesert G, Clarke DJ, Reed SI: Identification of genes controlling growth polarity in the budding yeast Saccharomyces cerevisiae: a possible role of $\mathbf{N}$-glycosylation and involvement of the exocyst complex. Genetics 1997, I 47:42I-34.

28. Dennis PB, Jaeschke A, Saitoh M, Fowler B, Kozma SC, Thomas G: Mammalian TOR: a homeostatic ATP sensor. Science 200I, 294: I 102-5.

29. Brachmann CB, Davies A, Cost GJ, Caputo E, Li J, Hieter P, Boeke JD: Designer deletion strains derived from Saccharomyces cerevisiae S288C: a useful set of strains and plasmids for PCRmediated gene disruption and other applications. Yeast 1998, 14:1 | 5-32.

30. Molk JN, Schuyler SC, Liu JY, Evans JG, Salmon ED, Pellman D, Bloom $\mathrm{K}$ : The differential roles of budding yeast TemIp, CdcI5p, and Bub2p protein dynamics in mitotic exit. Mol Biol Cell 2004, I5: $1519-32$

31. Dobbelaere J, Gentry MS, Hallberg RL, Barral Y: Phosphorylationdependent regulation of septin dynamics during the cell cycle. Dev Cell 2003, 4:345-57.

32. Sagot I, Bonneu M, Balguerie A, Aigle M: Imaging fluorescence resonance energy transfer between two green fluorescent proteins in living yeast. FEBS Lett 1999, 447:53-7.

33. Yang TT, Sinai P, Green G, Kitts PA, Chen YT, Lybarger L, Chervenak $\mathrm{R}$, Patterson GH, Piston DW, Kain SR: Improved fluorescence and dual color detection with enhanced blue and green variants of the green fluorescent protein. I Biol Chem 1998, 273:8212-6

34. Pinson B, Sagot I, Borne F, Gabrielsen OS, Daignan-Fornier B: Mutations in the yeast Myb-like protein Baslp resulting in discrimination between promoters in vivo but notin vitro. Nucleic Acids Res 1998, 26:3977-85.

35. Sagot I, Klee SK, Pellman D: Yeast formins regulate cell polarity by controlling the assembly of actin cables. Nat Cell Biol 2002, 4:42-50.

36. Breton AM, Schaeffer J, Aigle M: The yeast Rvs I6I and Rvs I67 proteins are involved in secretory vesicles targeting the plasma membrane and in cell integrity. Yeast 200।, I 8: 1053-68. 